TRANSACTIONS OF THE

AMERICAN MATHEMATICAL SOCIETY

Volume 361, Number 9, September 2009, Pages 5041-5060

S 0002-9947(09)04880-6

Article electronically published on April 16, 2009

\title{
SPECTRAL ANALYSIS \\ OF A CLASS OF NONLOCAL ELLIPTIC OPERATORS RELATED TO BROWNIAN MOTION WITH RANDOM JUMPS
}

\author{
ROSS G. PINSKY
}

\begin{abstract}
Let $D \subset R^{d}$ be a bounded domain and let $\mathcal{P}(D)$ denote the space of probability measures on $D$. Consider a Brownian motion in $D$ which is killed at the boundary and which, while alive, jumps instantaneously at an exponentially distributed random time with intensity $\gamma>0$ to a new point, according to a distribution $\mu \in \mathcal{P}(D)$. From this new point it repeats the above behavior independently of what has transpired previously. The generator of this process is an extension of the operator $-L_{\gamma, \mu}$, defined by

$$
L_{\gamma, \mu} u \equiv-\frac{1}{2} \Delta u+\gamma V_{\mu}(u)
$$

with the Dirichlet boundary condition, where $V_{\mu}$ is a nonlocal " $\mu$-centering" potential defined by

$$
V_{\mu}(u)=u-\int_{D} u d \mu .
$$

The operator $L_{\gamma, \mu}$ is symmetric only in the case that $\mu$ is normalized Lebesgue measure; thus, only in that case can it be realized as a selfadjoint operator. The corresponding semigroup is compact, and thus the spectrum of $L_{\gamma, \mu}$ consists exclusively of eigenvalues. As is well known, the principal eigenvalue gives the exponential rate of decay in $t$ of the probability of not exiting the domain by time $t$. We study the behavior of the eigenvalues, our main focus being on the behavior of the principal eigenvalue for the regimes $\gamma \gg 1$ and $\gamma \ll 1$. We also consider conditions on $\mu$ that guarantee that the principal eigenvalue is monotone increasing or decreasing in $\gamma$.
\end{abstract}

\section{InTRODUCTION AND STATEMENT OF RESULTS}

Let $D \subset R^{d}$ be a bounded domain with $C^{2, \alpha}$-boundary and let $\mathcal{P}(D)$ denote the space of probability measures on $D$. Fix a measure $\mu \in \mathcal{P}(D)$, and consider a Brownian motion in $D$ which is killed at the boundary and which, while alive, jumps instantaneously at an exponentially distributed random time with intensity $\gamma>0$ to a new point, according to the distribution $\mu$. From this new point it repeats the above behavior independently of what has transpired previously. Denote this process by $X(t)$, and let $\tau_{D}$ denote its lifetime. Denote probabilities and expectations for the Markov process $X(t)$ starting from $x \in D$ by $P_{x}^{\gamma, \mu}$ and $E_{x}^{\gamma, \mu}$.

Received by the editors June 18, 2007 and, in revised form, June 3, 2008.

2000 Mathematics Subject Classification. Primary 35P15, 60F10, 60J65.

Key words and phrases. Principal eigenvalue, spectral analysis, Brownian motion, random jumps.

This research was supported by the M. \& M. Bank Mathematics Research Fund.

(C)2009 American Mathematical Society Reverts to public domain 28 years from publication 
Define the contraction semigroup

$$
T_{t}^{\gamma, \mu} f(x)=E_{x}^{\gamma, \mu}\left(f(X(t)) ; \tau_{D}>t\right), f \in C_{0}(\bar{D}),
$$

where $C_{0}(\bar{D})$ is the space of continuous functions on $\bar{D}$ vanishing on $\partial D$. We will show that the infinitesimal generator of this semigroup is an extension of the operator $-L_{\gamma, \mu}$, defined on $C^{2}(\bar{D}) \cap\left\{u: u, L_{\gamma, \mu} u \in C_{0}(\bar{D})\right\}$ by

$$
L_{\gamma, \mu} u \equiv-\frac{1}{2} \Delta u+\gamma V_{\mu}(u)
$$

with the Dirichlet boundary condition, where $V_{\mu}$ is a nonlocal " $\mu$-centering" potential defined by

$$
V_{\mu}(u)=u-\int_{D} u d \mu
$$

We will show that the operator $T_{t}^{\gamma, \mu}$ is compact; thus, the resolvent operator for $T_{t}^{\gamma, \mu}$ is compact, and consequently the spectrum $\sigma\left(L_{\gamma, \mu}\right)$ of $L_{\gamma, \mu}$ consists exclusively of eigenvalues. By the Krein-Rutman theorem, one deduces that $L_{\gamma, \mu}$ possesses a principal eigenvalue, $\lambda_{0}(\gamma, \mu)$; that is, $\lambda_{0}(\gamma, \mu)$ is real and simple and satisfies $\lambda_{0}(\gamma, \mu)=\inf \left\{\operatorname{Re}(\lambda): \lambda \in \sigma\left(L_{\gamma, \mu}\right)\right\}$ [14. It is known that $\lambda \in \sigma\left(L_{\gamma, \mu}\right)$ if and only if $\exp (-\lambda t) \in \sigma\left(T_{t}^{\gamma, \mu}\right)$ [12. Thus, since $\left\|T_{t}^{\gamma, \mu}\right\|<1$, it follows that $\lambda_{0}(\gamma, \mu)>0$. We have

$$
\sup _{f \in C_{0}(\bar{D}),\|f\| \leq 1}\left\|T_{t}^{\gamma, \mu} f\right\|=\sup _{x \in D} P_{x}^{\gamma, \mu}\left(\tau_{D}>t\right) ;
$$

thus, a standard result [15] allows us to conclude that

$$
\lim _{t \rightarrow \infty} \frac{1}{t} \log \sup _{x \in D} P_{x}^{\gamma, \mu}\left(\tau_{D}>t\right)=-\lambda_{0}(\gamma, \mu) .
$$

It is well known that this is equivalent to

$$
\lim _{t \rightarrow \infty} \frac{1}{t} \log P_{x}^{\gamma, \mu}\left(\tau_{D}>t\right)=-\lambda_{0}(\gamma, \mu), \quad x \in D .
$$

The main focus in this paper is on the behavior of the principal eigenvalue for the regimes $\gamma \gg 1$ and $\gamma \ll 1$. We also consider conditions on $\mu$ that guarantee that the principal eigenvalue is monotone increasing or decreasing in $\gamma$.

The Brownian motion with random jumps analyzed here is a paradigm for a phenomenon that occurs in various settings and which is best illustrated perhaps in terms of computer games or the game "chutes and ladders". The object of the game is to reach the boundary of $D$ in as little time as possible (or alternatively, to avoid reaching the boundary for as much time as possible). The game is played in rounds; however, time is always accumulating. Various obstacles (modelled by the exponential clock with intensity $\gamma$ ) lead to the end of a round, and each new round begins afresh from a new position, which may be deterministic or random (modelled by the measure $\mu$ ). Then $\lambda_{0}(\gamma, \mu)$ is a measure of the probability of long-term failure (or success, depending on the rules).

A number of recent papers have treated Brownian motion with random jumps from the boundary, rather than from within the domain. Such a process is ergodic and possesses a unique invariant measure. The principal eigenvalue of the generator of the process is 0 , the rest of the spectrum is negative, and the spectral gap, which is the supremum of the real part of the nonzero spectrum, gives the exponential rate of convergence to equilibrium. See [1, [2], 8], 9], [11. 
In the past decade or so, a number of papers have treated spectral properties of elliptic operators with a nonlocal reaction term of the form

$$
(\nabla \cdot a \nabla u)(x)+b(x) u+c(x) \int_{D} d(y) u(y) d y,
$$

with the Dirichlet boundary condition, where $a$ is positive definite and $b, c$, and $d$ are functions. These papers study the location and multiplicities of the eigenvalues and the existence of a principal eigenvalue (this last point is automatic in our situation). See, for example, 4, , 5], 3] and the references therein for results and applications. See also Remark 3 after Theorem 3 and Remark 1 after Theorem 4 .

We now turn to the results, considering first the regime $\gamma \gg 1$. Before stating the theorem, we note that probabilistic intuition suggests the general direction of the result. Since $\gamma \gg 1$, the Brownian motion doesn't get very far before it jumps and gets redistributed according to $\mu$. In particular then, if $\operatorname{supp}(\mu) \subset D$, it will be very difficult for the Brownian motion to exit $D$, and in light of (1.1) one expects that $\lim _{\gamma \rightarrow \infty} \lambda_{0}(\gamma, \mu)=0$. More generally, one expects that the leading asymptotic behavior for large $\gamma$ will depend only on the behavior of $\mu$ arbitrarily close to the boundary.

For $\epsilon>0$, let $D^{\epsilon}=\{x \in D: \operatorname{dist}(x, \partial D)<\epsilon\}$. We will prove the following result.

Theorem 1. Let $D \subset R^{d}, d \geq 1$, be a bounded domain and let $\mu \in \mathcal{P}(D)$.

$i$. Assume that for some $\epsilon>0$, the restriction of $\mu$ to $D^{\epsilon}$ possesses a density which belongs to $C\left(\bar{D}^{\epsilon}\right):\left.\mu(d x)\right|_{D^{\epsilon}} \equiv \mu(x) d x$. Then

$$
\lim _{\gamma \rightarrow \infty} \gamma^{-\frac{1}{2}} \lambda_{0}(\gamma, \mu)=\frac{1}{\sqrt{2}} \int_{\partial D} \mu d \sigma
$$

ii. Assume that for some $\epsilon>0$, the restriction of $\mu$ to $D^{\epsilon}$ possesses a density which belongs to $C_{b}^{2}\left(D^{\epsilon}\right) \cap C^{1}\left(\bar{D}^{\epsilon}\right)$ and vanishes on $\partial D:\left.\mu(d x)\right|_{D^{\epsilon}} \equiv \mu(x) d x$. Then

$$
\lim _{\gamma \rightarrow \infty} \lambda_{0}(\gamma, \mu)=\frac{1}{2} \int_{\partial D}(\nabla \mu \cdot n) d \sigma
$$

where $n$ is the inward unit normal vector on $\partial D$.

iii. Assume that for some $\epsilon>0$, the restriction of $\mu$ to $D^{\epsilon}$ possesses a density which belongs to $C_{b}^{2}\left(\bar{D}^{\epsilon}\right)$ and is such that $\mu$ and $\nabla \mu$ vanish on $\partial D:\left.\mu(d x)\right|_{D^{\epsilon}} \equiv$ $\mu(x) d x$. Then

$$
\lim _{\gamma \rightarrow \infty} \gamma^{\frac{1}{2}} \lambda_{0}(\gamma, \mu)=\frac{1}{2 \sqrt{2}} \int_{\partial D} \Delta \mu d \sigma .
$$

(One has $\Delta \mu \geq 0$ on $\partial D$ since $\mu$ and $\nabla \mu$ vanish on $\partial D$ and $\mu$ is nonnegative in D.)

iv. Assume that $\mu \in \mathcal{P}(D)$ is compactly supported. Then

$$
\lim _{\gamma \rightarrow \infty} \lambda_{0}(\gamma, \mu)=0
$$

In fact, letting $l=\operatorname{dist}(\operatorname{supp}(\mu), \partial D)$ and $a=\sup \{|x-z|: z \in \partial D, x \in \operatorname{supp}(\mu)\}$, there exists a constant $c_{l, d}$ such that

$$
\frac{\gamma}{1+\left(\frac{a^{2} \pi^{2} \gamma}{2}\right)^{\frac{1}{4}}} \exp \left(-\sqrt{2} a \gamma^{\frac{1}{2}}\right)<\lambda_{0}(\gamma, \mu)<(2 d+1) \gamma \exp \left(-\frac{l \gamma^{\frac{1}{2}}}{2 \sqrt{2} d}\right), \text { for } \gamma \geq c_{l, d}
$$


(In parts (i)-(iii), $\sigma$ denotes Lebesgue measure on $\partial D$ if $d \geq 2$, while it is the counting measure on the endpoints of $D$ if $d=1$.)

Remark. We expect that if $\mu$ and all its partial derivatives up to order $k$ vanish on $\partial D$, and the derivatives of order $k+1$ do not all vanish identically on $\partial D$, then $\lambda_{0}(\gamma, \mu)$ will decay on the order of $\gamma^{-\frac{k}{2}}$. Similarly, if the density is allowed to blow up at the boundary, then the order $\gamma^{\frac{1}{2}}$ in part (i) will increase. By Proposition 1 in Section 2, the order can never be greater than $\gamma$.

Example. When $\mu=l_{D}$, the normalized Lebesgue measure on $D$, Theorem 1 gives

$$
\lim _{\gamma \rightarrow \infty} \gamma^{-\frac{1}{2}} \lambda_{0}\left(\gamma, l_{D}\right)=\frac{1}{\sqrt{2}} \frac{|\partial D|}{|D|} .
$$

We now turn to the regime $\gamma \ll 1$. Of course, $\lambda_{0}(0, \mu)=\lambda_{0}^{D}$, where $\lambda_{0}^{D}$ is the principal eigenvalue of $-\frac{1}{2} \Delta$ in $D$ with the Dirichlet boundary condition. (Henceforth, this operator (with the negative sign) will be referred to as the Dirichlet Laplacian.) We wish to determine when $\lambda_{0}(\gamma, \mu)>\lambda_{0}^{D}$ and when $\lambda_{0}(\gamma, \mu)<\lambda_{0}^{D}$, for small $\gamma$. In the former (latter) case, random jumps at low intensity cause the probability of the event $\left\{\tau_{D}>t\right\}$ to decay more (less) rapidly than it would for standard Brownian motion without random jumps. Let $\phi_{0}$ denote the principal eigenfunction, normalized by $\phi_{0}>0$ and $\int_{D} \phi_{0}^{2} d x=1$, corresponding to the principal eigenvalue $\lambda_{0}^{D}$ for the Dirichlet Laplacian. Let

$$
F_{0} \equiv \int_{D} \phi_{0} d x \text { and } G_{0}(\mu) \equiv \int_{D} \phi_{0} d \mu .
$$

Let $V_{0}$ denote the solution to the equation

$$
\begin{aligned}
& \frac{1}{2} \Delta V+\lambda_{0}^{D} V=-1+F_{0} \phi_{0} \text { in } D ; \\
& V=0 \text { on } \partial D ; \\
& \int_{D} V \phi_{0} d x=0 .
\end{aligned}
$$

(Since $\left(\frac{1}{2} \Delta+\lambda_{0}^{D}\right) \phi_{0}=0$ and $\int_{D}\left(-1+F_{0} \phi_{0}\right) \phi_{0} d x=0$, the Fredholm alternative guarantees the existence of a unique solution to (1.4).)

Theorem 2. $i$.

$$
\frac{d \lambda_{0}}{d \gamma}\left(0^{+}, \mu\right)=1-F_{0} G_{0}(\mu)=1-\int_{D} \phi_{0} d x \int_{D} \phi_{0} d \mu .
$$

Thus, if $F_{0} G_{0}(\mu)<1\left(F_{0} G_{0}(\mu)>1\right)$, then $\lambda_{0}(\gamma, \mu)>\lambda_{0}^{D}\left(\lambda_{0}(\gamma, \mu)<\lambda_{0}^{D}\right)$, for $\gamma \ll 1$.

ii. If $F_{0} G_{0}(\mu)=1$ and $\int_{D} V_{0} d \mu<0\left(\int_{D} V_{0} d \mu>0\right)$, then $\lambda_{0}(\gamma, \mu)>\lambda_{0}^{D}$ $\left(\lambda_{0}(\gamma, \mu)<\lambda_{0}^{D}\right)$, for $\gamma \ll 1$, where $V_{0}$ is the solution to (1.4).

Example 1. Consider the case that $\mu=l_{D}$, the normalized Lebesgue measure on $D$. One has $G_{0}\left(l_{D}\right)=\frac{F_{0}}{|D|}$. The Cauchy-Schwarz inequality gives $F_{0} G_{0}\left(l_{D}\right)=$ $\frac{1}{|D|}\left(\int_{D} \phi_{0} d x\right)^{2}<\int_{D} \phi_{0}^{2} d x=1$, and thus by Theorem $2, \lambda_{0}\left(\gamma, l_{D}\right)>\lambda_{0}^{D}$, for $\gamma \ll 1$.

Example 2. Consider the case $D=(0,1)$ with $\mu=\delta_{x_{0}}$, for some $x_{0} \in(0,1)$. In this case, we have $\phi_{0}(x)=\sqrt{2} \sin \pi x$, so $F_{0}=\frac{2 \sqrt{2}}{\pi}$ and $G_{0}=\sqrt{2} \sin \pi x_{0}$. Let $x_{c} \equiv \frac{1}{\pi} \arcsin \frac{\pi}{4} \approx .288$. Then $F_{0} G_{0}\left(\delta_{x_{0}}\right)<1$ if and only if $x_{0} \in\left(0, x_{c}\right) \cup\left(1-x_{c}, 1\right)$. 
Consider now the borderline case, $\mu=\delta_{x_{c}}$. A long and tedious calculation reveals that the solution $V_{0}$ to (1.4) is given by $V_{0}(x)=\frac{6}{\pi^{3}} \sin \pi x+\frac{2}{\pi^{2}} \cos \pi x-\frac{2}{\pi^{2}}-$ $\frac{4}{\pi^{2}} x \cos \pi x$. One checks that $\int_{0}^{1} V_{0}(x) d \mu=V_{0}\left(x_{c}\right)>0$. Thus, we conclude from Theorem 2 that for $\gamma \ll 1, \lambda_{0}\left(\gamma, \delta_{x_{0}}\right)$ is greater than $\lambda_{0}^{D}$ if $x_{0} \in\left(0, x_{c}\right) \cup\left(1-x_{c}, 1\right)$ and is less than $\lambda_{0}^{D}$ if $x_{0} \in\left[x_{c}, 1-x_{c}\right]$.

It follows from Theorems 1 and 2 that $\lambda_{0}(\gamma, \mu)$ is frequently not monotone in $\gamma$; one can easily construct examples where it increases and then decreases or vice versa. Note that if there exists a point $x_{0} \in D$ such that $\mathcal{P}_{x_{0}}\left(\tau_{D}>t\right) \geq \mathcal{P}_{x}\left(\tau_{D}>\right.$ $t$ ), for all $x \in D$ and all $t>0$, where $\mathcal{P}_{x}\left(\tau_{D}>t\right)$ denotes the probability that a standard Brownian motion starting from $x \in D$ has not exited $D$ by time $t$, then by the definition of the Brownian motion with random jumps along with probabilistic considerations and (1.1), it follows that $\lambda_{0}\left(\gamma, \delta_{x_{0}}\right)$ is decreasing in $\gamma$, and furthermore, that $\lambda_{0}\left(\gamma, \delta_{x_{0}}\right) \leq \lambda_{0}(\gamma, \mu)$, for all $\mu \in \mathcal{P}(D)$ and all $\gamma>0$. In particular, this occurs if $D=(0,1)$ and $x_{0}=\frac{1}{2}$.

In the case that $\mu=l_{D}$, the normalized Lebesgue measure on $D, L_{\gamma, \mu}$ is symmetric and can be realized as a selfadjoint operator. We can express the corresponding quadratic form as

$$
\int_{D} u L_{\gamma, \mu} u d x=\frac{1}{2} \int_{D}|\nabla u|^{2} d x+\gamma|D| \operatorname{Var}_{l_{D}}(u),
$$

where $\operatorname{Var}_{l_{D}}(u)=\int_{D} u^{2} d l_{D}-\left(\int_{D} u d l_{D}\right)^{2} \geq 0$ is the variance of $u$ with respect to the probability measure $l_{D}$, and $u \in C^{2}(D) \cap C_{0}(\bar{D})$. Thus, $\lambda_{0}(\gamma, \mu)$ is given by the variational formula

$$
\lambda_{0}(\gamma, \mu)=\inf _{0 \neq u \in C^{2}(D) \cap C_{0}(\bar{D})} \frac{\frac{1}{2} \int_{D}|\nabla u|^{2} d x+\gamma|D| \operatorname{Var}_{l_{D}}(u)}{\int_{D} u^{2} d x} .
$$

From this it follows that $\lambda_{0}(\gamma, \mu)$ is strictly monotone increasing in $\gamma$. Because of the selfadjointness, it also follows that all of the eigenvalues of $L_{\gamma, \mu}$ are real.

In fact, we can single out two classes of measures $\mu$, each defined by a spectraltheoretic condition, for one of which $\lambda_{0}(\gamma, \mu)$ is monotone increasing and for the other of which it is monotone decreasing, and for both of which all of the eigenvalues are real, even though $L_{\gamma, \mu}$ is not selfadjoint when $\mu \neq l_{D}$.

We will need some additional notation to state the result. We have already introduced $\lambda_{0}^{D}$ and $\phi_{0}$. Let $\left\{\lambda_{n}^{D}\right\}_{n=1}^{\infty}$ denote all the nonprincipal eigenvalues of the Dirichlet Laplacian, labelled in increasing order, and let $\left\{\phi_{n}\right\}_{n=1}^{\infty}$ denote the corresponding eigenfunctions, normalized by $\int_{D} \phi_{n}^{2} d x=1$. Let

$$
F_{n} \equiv \int_{D} \phi_{n} d x \text { and } G_{n}(\mu) \equiv \int_{D} \phi_{n} d \mu
$$

When $d \geq 3$, we will sometimes need to assume that the domain $D$ satisfies the following condition.

Assumption 1. $\sum_{n=0}^{\infty} \frac{F_{n}}{\lambda_{n}^{D}} \phi_{n}$ converges uniformly and absolutely on $D$.

Remark. When $d=1,2$, Assumption 1 always holds [2, 7]. One has $\sum_{n=0}^{\infty} \frac{1}{\left(\lambda_{n}^{D}\right)^{2}}<$ $\infty$ if and only if $d \leq 3\left[2\right.$. Thus, since $\left\{F_{n}\right\} \in l_{2}$, Assumption 1 holds when $d=3$ if the $\phi_{n}$ are uniformly bounded.

Let $\left\{\Lambda_{n}^{D}\right\}_{n=0}^{\infty}$ denote the collection of distinct values among the eigenvalues $\left\{\lambda_{n}^{D}\right\}_{n=0}^{\infty}$ of the Dirichlet Laplacian, labelled in increasing order. Let $P_{\Lambda_{n}^{D}}$ denote 
the orthogonal projection onto the eigenspace corresponding to the eigenvalue $\Lambda_{n}^{D}$. Note that

and that

$$
\int_{D} P_{\Lambda_{0}^{D}} 1 d \mu=F_{0} G_{0}(\mu)>0
$$

$$
\int_{D} P_{\Lambda_{n}^{D}} 1 d \mu=\sum_{m: \lambda_{m}^{D}=\Lambda_{n}^{D}} F_{m} G_{m}(\mu) .
$$

Theorem 3. Let $\mu \in \mathcal{P}(D)$. For part (i), assume that $\mu$ possesses an $L^{2}$-density. For parts (ii) and (iii), if $d \geq 3$ assume either that $\mu$ possesses an $L^{2}$-density or that the domain $D$ satisfies Assumption 1 .

$i$-a. If $\int_{D} P_{\Lambda_{n}^{D}} 1 d \mu=\sum_{m: \lambda_{m}^{D}=\Lambda_{n}^{D}} F_{m} G_{m}(\mu)=0$, for all $n \geq 1$, then $\lambda_{0}(\gamma, \mu)=$ $\lambda_{0}^{D}$, for all $\gamma>0$.

$i$-b. If $\int_{D} P_{\Lambda_{n}^{D}} 1 d \mu=\sum_{m: \lambda_{m}^{D}=\Lambda_{n}^{D}} F_{m} G_{m}(\mu) \geq 0$, for all $n \geq 1$, and is nonzero for at least one value of $n \geq 1$, then $\lambda_{0}(\gamma, \mu)$ is strictly increasing in $\gamma$.

$i$-c. If $\int_{D} P_{\Lambda_{n}^{D}} 1 d \mu=\sum_{m: \lambda_{m}^{D}=\Lambda_{n}^{D}} F_{m} G_{m}(\mu) \leq 0$, for all $n \geq 1$, and is nonzero for at least one value of $n \geq 1$, then $\lambda_{0}(\gamma, \mu)$ is strictly decreasing in $\gamma$.

ii. If $\int_{D} P_{\Lambda_{n}^{D}} 1 d \mu=\sum_{m: \lambda_{m}^{D}=\Lambda_{n}^{D}} F_{m} G_{m}(\mu)$ is nonnegative for all $n \geq 1$ or nonpositive for all $n \geq 1$, then all of the eigenvalues of $L_{\gamma, \mu}$ are real.

iii. Assume that $\int_{D} P_{\Lambda_{n}^{D}} 1 d \mu=F_{n} G_{n}(\mu)>0$, for all $n \geq 1$, and assume that all the eigenvalues of the Dirichlet Laplacian are distinct. Thus, $\lambda_{n}^{D}=\Lambda_{n}^{D}$ and $\int_{D} P_{\Lambda_{n}^{D}} 1 d \mu=F_{n} G_{n}(\mu)$. Then

$$
\gamma+\lambda_{n-1}^{D}<\lambda_{n}(\gamma, \mu)<\gamma+\lambda_{n}^{D}, n \geq 1
$$

Remark 1. Recall that the function 1 is represented in $L^{2}$ by $\sum_{n=0}^{\infty} F_{n} \phi_{n}$. The assumption that $\mu$ has an $L^{2}$-density is used in the proof of part (i) in order to guarantee that $\sum_{n=0}^{\infty} F_{n} G_{n}(\mu)=1$. (In fact, one can check that the proof of part (i-a) goes through as long as $\sum_{n=0}^{\infty} F_{n} G_{n}(\mu) \leq 1$ and the proof of part (i-b) goes through as long as $\sum_{n=0}^{\infty} F_{n} G_{n}(\mu) \geq 1$.) If $\sum_{n=0}^{\infty} F_{n} \phi_{n}$ converges boundedly pointwise on the support of $\mu$, then the bounded convergence theorem gives $\sum_{n=0}^{\infty} F_{n} G_{n}(\mu)=1$, and thus part (i) holds in such a case even if $\mu$ does not have an $L^{2}$-density. In the one-dimensional case, one can show that $\sum_{n=0}^{\infty} F_{n} \phi_{n}$ converges boundedly pointwise away from the endpoints. Thus, when $d=1$, part (i) can be applied to any compactly supported $\mu$. Of course, such a $\mu$ cannot satisfy the condition of part (i-a) or (i-b) since if it did, then $\lambda_{0}(\gamma, \mu)$ would be nondecreasing in $\gamma$, contradicting Theorem [1]

Remark 2. The conditions for monotonicity in part (i) are of course only sufficient. When $D=(0,1)$ and $\mu=\delta_{\frac{1}{2}}$, then as noted earlier, $\lambda_{0}\left(\gamma, \delta_{\frac{1}{2}}\right)$ is decreasing in $\gamma$; however $F_{2 n} G_{2 n}\left(\delta_{\frac{1}{2}}\right)=\frac{4}{(2 n+1) \pi} \sin \frac{2 n+1}{2} \pi$, which alternates sign.

Remark 3. In the one-dimensional setting, part (ii) of the theorem was proved in 4].

Remark 4. When one (or both) of the assumptions appearing in the statement of part (iii) is violated, one can still use the method of proof there to give estimates on the eigenvalues; however each particular case must be treated separately.

The proofs of Theorems 1 and 2 utilize a characterization of the principal eigenvalue which appears in Proposition 1 in Section 2. This characterization does not 
allow for a proof of Theorem 3 . Instead, we make use of the following characterization of all of the eigenvalues of $L_{\gamma, \mu}$, which is of some interest in its own right.

Theorem 4. Let $\mu \in \mathcal{P}(D)$ and $\gamma>0$. If $d \geq 3$, assume either that $\mu$ possesses an $L^{2}$-density or that the domain $D$ satisfies Assumption 1 , Let $d_{n}$ denote the dimension of the eigenspace corresponding to the $n$-th distinct eigenvalue $\Lambda_{n}^{D}$ of the Dirichlet Laplacian. Let

$$
E_{\gamma, \mu}(\lambda) \equiv \sum_{n=0}^{\infty} \frac{\gamma F_{n} G_{n}(\mu)}{\gamma+\lambda_{n}^{D}-\lambda}=\sum_{n=0}^{\infty} \frac{\gamma \int_{D} P_{\Lambda_{n}^{D}} 1 d \mu}{\gamma+\Lambda_{n}^{D}-\lambda}
$$

The set of eigenvalues of $L_{\gamma, \mu}$ and their multiplicities are given as follows.

$i$. The set $\left\{\lambda: E_{\gamma, \mu}(\lambda)=1\right\} \backslash\left\{\gamma+\Lambda_{n}^{D}\right\}_{n=1}^{\infty}$ consists of simple eigenvalues.

ii. For each $n=1,2, \ldots$, the following rule determines whether $\gamma+\Lambda_{n}^{D}$ is an eigenvalue, and if so, specifies its multiplicity.

If $d_{n}=1$ and neither $F_{m}=0$ nor $G_{m}(\mu)=0$, for the unique $m$ satisfying $\lambda_{m}^{D}=\Lambda_{n}^{D}$, then $\gamma+\Lambda_{n}^{D}$ is not an eigenvalue. Otherwise, $\gamma+\Lambda_{n}^{D}$ is an eigenvalue and its multiplicity is specified as follows:

if $G_{m}(\mu) \neq 0$ for some $m$ such that $\lambda_{m}^{D}=\Lambda_{n}^{D}$ and $F_{m} \neq 0$ for some $m$ such that $\lambda_{m}^{D}=\Lambda_{n}^{D}$, then the multiplicity is $d_{n}-1$;

if $G_{m}(\mu)=0$ for all $m$ such that $\lambda_{m}^{D}=\Lambda_{n}^{D}$ and $F_{m} \neq 0$ for some $m$ such that $\lambda_{m}^{D}=\Lambda_{n}^{D}$, or if $G_{m}(\mu) \neq 0$ for some $m$ such that $\lambda_{m}^{D}=\Lambda_{n}^{D}$ and $F_{m}=0$ for all $m$ such that $\lambda_{m}^{D}=\Lambda_{n}^{D}$, then the multiplicity is $d_{n}$;

if $G_{m}(\mu)=0$ for all $m$ such that $\lambda_{m}^{D}=\Lambda_{n}^{D}$ and $F_{m}=0$ for all $m$ such that $\lambda_{m}^{D}=$ $\Lambda_{n}^{D}$, then the multiplicity is $d_{n}$ if $E_{\gamma, \mu}\left(\gamma+\Lambda_{n}^{D}\right) \neq 0$ and is $d_{n+1}$ if $E_{\gamma, \mu}\left(\gamma+\Lambda_{n}^{D}\right)=0$.

Remark 1. Partial results along the lines of Theorem 4 can be found in [5] and [3].

Remark 2. For $n \geq 0$, if $\int_{D} P_{\Lambda_{n}^{D}} 1 d \mu$ and $\int_{D} P_{\Lambda_{n+1}^{D}} 1 d \mu$ have opposite signs, then there is an eigenvalue between $\gamma+\Lambda_{n}^{D}$ and $\gamma+\Lambda_{n+1}^{D}$, since in this case $E_{\gamma, \mu}(\lambda)$, with $\Lambda_{n}^{D}<\lambda<\Lambda_{n+1}^{D}$, approaches $+\infty$ as $\lambda$ approaches one endpoint and approaches $-\infty$ as $\lambda$ approaches the other endpoint.

The rest of the paper is organized as follows. In Section 2 we prove a couple of preliminary results concerning the principle eigenvalue $\lambda_{0}(\gamma, \mu)$, which will be used to prove Theorems 1 and 2, the proofs of which are given in Sections 3 and 4. respectively. We prove Theorem 4 in Section 5 and Theorem 3 , whose proof depends on Theorem 4, in Section 6. In an appendix we show that $-L_{\gamma, \mu}$, suitably extended, is the infinitesimal generator of $T_{t}^{\gamma, \mu}$, and that $T_{t}^{\gamma, \mu}$ is compact.

All the results in the paper go through with only cosmetic changes when Brownian motion and the Laplacian are replaced by a general reversible diffusion and its generator $A \equiv \frac{1}{2} \exp (-2 Q) \nabla \cdot \exp (2 Q) a \nabla=\frac{1}{2} \nabla \cdot a \nabla+\nabla Q \nabla$.

\section{Preliminary Results for the Principal eigenvalue}

Let $Y(t)$ denote the standard Brownian motion in $D$ without jumps, which is killed at the boundary, let $\tau_{D}$ denote its lifetime and denote the corresponding probabilities and expectations by $\mathcal{P}$. and $\mathcal{E}$. 
For $c \in\left(-\infty, \lambda_{0}^{D}\right)$, let $w_{c}>0$ denote the solution to the equation

$$
\begin{aligned}
& \frac{1}{2} \Delta w+c w=-1 \text { in } D ; \\
& w=0 \text { on } \partial D .
\end{aligned}
$$

Applying Ito's formula with the stopping time $\tau_{D}$ gives

$$
\mathcal{E}_{x} \exp \left(c \tau_{D}\right) w_{c}\left(Y\left(\tau_{D}\right)\right)=w_{c}(x)+\mathcal{E}_{x} \int_{0}^{\tau_{D}}\left(\frac{1}{2} \Delta+c\right) w_{c}(Y(t)) \exp (c t) d t .
$$

From (2.1) and (2.2) we obtain

$$
w_{c}(x)=\left\{\begin{array}{l}
\frac{1}{c}\left(\mathcal{E}_{x} \exp \left(c \tau_{D}\right)-1\right), \text { if } c \neq 0 \\
\mathcal{E}_{x} \tau_{D}, \text { if } c=0 .
\end{array}\right.
$$

(The condition $c<\lambda_{0}^{D}$ is necessary and sufficient for the existence of a positive solution to (2.1) and for the finiteness of $\mathcal{E}_{x} \exp \left(c \tau_{D}\right)$ [14, Chapter 3].) Similarly, the solution $u_{c}$ to the equation

$$
\begin{aligned}
& \frac{1}{2} \Delta u+c u=0 \text { in } D, \\
& u=1 \text { on } \partial D,
\end{aligned}
$$

is given by

$$
u_{c}(x)=\mathcal{E}_{x} \exp \left(c \tau_{D}\right) .
$$

We begin with a characterization of the principal eigenvalue $\lambda_{0}(\gamma, \mu)$ of $L_{\gamma, \mu}$.

Proposition 1. One has $\lambda_{0}(\gamma, \mu)<\gamma+\lambda_{0}^{D}$, for all $\gamma>0$. More specifically, for $\lambda<\gamma+\lambda_{0}^{D}$, consider the equation

$$
\lambda=\left\{\begin{array} { l } 
{ \gamma \mathcal { E } _ { \mu } \operatorname { e x p } ( ( \lambda - \gamma ) \tau _ { D } ) , \gamma \neq \lambda } \\
{ ( \mathcal { E } _ { \mu } \tau _ { D } ) ^ { - 1 } , \gamma = \lambda }
\end{array} \quad \left(\text { or equivalently, } \lambda=\left\{\begin{array}{l}
\gamma \int_{D} u_{\lambda-\gamma} d \mu, \gamma \neq \lambda \\
\left(\mathcal{E}_{\mu} \tau_{D}\right)^{-1}, \gamma=\lambda
\end{array}\right) .\right.\right.
$$

i. If $\gamma>\left(\mathcal{E}_{\mu} \tau_{D}\right)^{-1}$, then $\lambda_{0}(\gamma, \mu) \in(0, \gamma)$ and is equal to the smallest root $\lambda \in(0, \gamma)$ of (2.6).

ii. If $\gamma<\left(\mathcal{E}_{\mu} \tau_{D}\right)^{-1}$, then $\lambda_{0}(\gamma, \mu) \in\left(\gamma, \gamma+\lambda_{0}^{D}\right)$ and is equal to the smallest root $\lambda \in\left(\gamma, \gamma+\lambda_{0}^{D}\right)$ of (2.6).

iii. If $\gamma=\left(\mathcal{E}_{\mu} \tau_{D}\right)^{-1}$, then $\lambda_{0}(\gamma, \mu)=\gamma=\left(\mathcal{E}_{\mu} \tau_{D}\right)^{-1}$.

Proof. Let $w$ denote the principal eigenfunction corresponding to $\lambda_{0}(\gamma, \mu)$, normalized by $\int_{D} w d \mu=1$. Then

$$
\begin{aligned}
& \frac{1}{2} \Delta w-\gamma w+\gamma=-\lambda_{0}(\gamma, \mu) w \text { in } D \\
& w=0 \text { on } \partial D .
\end{aligned}
$$

Thus, $w=\gamma w_{\lambda_{0}(\gamma, \mu)-\gamma}$. From the normalization condition above, it then follows that $\lambda=\lambda_{0}(\gamma, \mu)$ is a solution to the equation

$$
\gamma \int_{D} w_{\lambda-\gamma} d \mu=1
$$

Conversely, if $\lambda$ solves (2.7), then it is an eigenvalue. Consequently, $\lambda_{0}(\gamma, \mu)$ is the smallest solution $\lambda$ to (2.7). By (2.3) and (2.5), it follows that (2.7) is equivalent to (2.6). 
Fix $\gamma>0$ and let $q(\lambda)=\lambda-\gamma \mathcal{E}_{\mu} \exp \left((\lambda-\gamma) \tau_{D}\right)$. Then $q(0)<0$ and $q(\gamma)=0$. If $\gamma>\left(\mathcal{E}_{\mu} \tau_{D}\right)^{-1}$, then $q^{\prime}(\gamma)<0$, and we conclude that the smallest root $\lambda$ of (2.6) occurs in $(0, \gamma)$. This proves part (i). If $\gamma \leq\left(\mathcal{E}_{\mu} \tau_{D}\right)^{-1}$, then $q^{\prime}(\lambda)>0$, for $\lambda \in(0, \gamma)$, and thus (2.6) has no root $\lambda \in(0, \gamma)$. If $\gamma=\left(\mathcal{E}_{\mu} \tau_{D}\right)^{-1}$, we then conclude that the smallest root of (2.6) occurs at $\lambda=\gamma$. If $\gamma<\left(\mathcal{E}_{\mu} \tau_{D}\right)^{-1}$, then $q^{\prime}(\gamma)>0$. Since $q\left(\gamma+\lambda_{0}^{D}\right)=-\infty$, we conclude that the smallest root of (2.6) occurs in $\left(\gamma, \gamma+\lambda_{0}^{D}\right)$.

The following lemma will be used repeatedly.

\section{Lemma 1.}

$$
\lim _{\gamma \rightarrow \infty} \frac{\lambda_{0}(\gamma, \mu)}{\gamma}=0
$$

Proof. By Proposition 10 it is enough to show that as $\gamma \rightarrow \infty$, the quotient $\frac{\lambda_{0}(\gamma, \mu)}{\gamma}$ has no accumulation points in $(0,1]$. We first show that there are no accumulation points in $(0,1)$. If $p \in(0,1)$ were an accumulation point, then substituting $\lambda_{0}(\gamma, \mu)$ for $\lambda$ in (2.6), dividing both sides by $\gamma$ and letting $\gamma \rightarrow \infty$ along an appropriate sequence would give the contradiction $p=0$.

We now show that 1 is not an accumulation point. Assume to the contrary that $\lim _{n \rightarrow \infty} \frac{\lambda_{0}\left(\gamma_{n} \cdot \mu\right)}{\gamma_{n}}=1$, where $\lim _{n \rightarrow \infty} \gamma_{n}=\infty$. By Proposition 11, we have $\lambda_{0}\left(\gamma_{n}, \mu\right)=\gamma_{n}-c_{n}$, where $c_{n}>0$ for sufficiently large $n$. Without loss of generality we may assume that $\lim _{n \rightarrow \infty} c_{n} \equiv c_{\infty} \in[0, \infty]$ exists. First consider the case that $c_{\infty} \neq 0$. Then substituting $\lambda_{0}\left(\gamma_{n}, \mu\right), \gamma_{n}$ and $-c_{n}$ respectively for $\lambda, \gamma$ and $\lambda-\gamma$ in (2.6), dividing both sides by $\gamma_{n}$ and letting $n \rightarrow \infty$ gives the contradiction $1=\mathcal{E}_{\mu} \exp \left(-c_{\infty} \tau_{D}\right)$. Now consider the case $c_{\infty}=0$. Rewrite (2.6) as $\lambda-\gamma=\gamma\left(\mathcal{E}_{\mu} \exp \left((\lambda-\gamma) \tau_{D}\right)-1\right)$. Substituting as before, we obtain $-c_{n}=\gamma_{n}\left(\mathcal{E}_{\mu} \exp \left(-c_{n} \tau_{D}\right)-1\right)$. Dividing both sides by $-c_{n}$ and letting $n \rightarrow \infty$, we obtain the contradiction $1=\infty$.

\section{Proof of Theorem 1}

It will be convenient to prove the results in an order different from that in which they were stated.

Part iv. Clearly $\mathcal{E}_{\mu} \tau_{D}$ can be bounded from below by a positive constant depending only on $l \equiv \operatorname{dist}(\operatorname{supp}(\mu), \partial D)$ and $d$. By Proposition 1, $\lambda_{0}(\gamma, \mu)$ is the smallest root $\lambda \in(0, \gamma)$ of (2.6) when $\gamma>\left(\mathcal{E}_{\mu} \tau_{D}\right)^{-1}$. As functions of $\lambda$, both the left hand side and the right hand side of (2.6) are increasing. Furthermore, the left hand side is smaller than the right hand side when $\lambda=0$, and it is larger than the right hand side when $\lambda=\frac{\gamma}{2}$, if $\mathcal{E}_{\mu} \exp \left(-\frac{\gamma}{2} \tau_{D}\right)<\frac{1}{2}$. This last inequality holds when $\gamma$ is larger than a constant depending only on $l$ and $d$. Thus there exists a constant $c_{l, d}$ such that

$$
\gamma \int_{D} \mathcal{E}_{x} \exp \left(-\gamma \tau_{D}\right) d \mu(x)<\lambda_{0}(\gamma, \mu)<\gamma \int_{D} \mathcal{E}_{x} \exp \left(-\frac{1}{2} \gamma \tau_{D}\right) d \mu(x), \text { for } \gamma \geq c_{l, d} .
$$

One has the following inequality [14, Chapter 2]:

$$
\mathcal{P}_{x}\left(\tau_{D} \leq t\right) \leq 2 d \exp \left(-\frac{\operatorname{dist}(x, \partial D)^{2}}{2 d t}\right) .
$$


Letting $t=\sqrt{\frac{2}{\gamma}} l$ in $(\underline{3.2})$, we have the estimate

$$
\begin{aligned}
& \mathcal{E}_{x} \exp \left(-\frac{1}{2} \gamma \tau_{D}\right) \leq \mathcal{P}_{x}\left(\tau_{D} \leq t\right)+\exp \left(-\frac{1}{2} \gamma t\right) \\
& <2 d \exp \left(-\frac{l \gamma^{\frac{1}{2}}}{2 \sqrt{2} d}\right)+\exp \left(-\frac{l \gamma^{\frac{1}{2}}}{\sqrt{2}}\right), \text { if } \operatorname{dist}(x, \partial D) \geq l .
\end{aligned}
$$

Thus, the upper bound in (1.2) follows from (3.3) and (3.1).

By the reflection principle for one-dimensional Brownian motion [10, it follows that in any dimension,

$$
\mathcal{P}_{x}\left(\tau_{D} \leq t\right) \geq 2 \int_{a}^{\infty} \frac{1}{(2 \pi t)^{\frac{1}{2}}} \exp \left(-\frac{|y|^{2}}{2 t}\right) d y, \text { where } a=\sup \{|x-z|: z \in \partial D\}
$$

One has the following inequality [13, Lemma 3.6]:

$$
\begin{aligned}
& \int_{a}^{\infty} \frac{1}{(2 \pi t)^{\frac{1}{2}}} \exp \left(-\frac{|y|^{2}}{2 t}\right) d y=\int_{a t^{-\frac{1}{2}}}^{\infty} \frac{1}{(2 \pi)^{\frac{1}{2}}} \exp \left(-\frac{|y|^{2}}{2}\right) d y \\
& \geq \frac{1}{(2 \pi)^{\frac{1}{2}}} \frac{1}{a t^{-\frac{1}{2}}+\left(\frac{2}{\pi}\right)^{\frac{1}{2}}} \exp \left(-\frac{a^{2}}{2 t}\right) .
\end{aligned}
$$

Letting $t=\frac{a}{(2 \gamma)^{\frac{1}{2}}}$ in (3.5), and using (3.4), we have the estimate

$$
\begin{aligned}
& \mathcal{E}_{x} \exp \left(-\gamma \tau_{D}\right) \geq \exp (-\gamma t) \mathcal{P}_{x}\left(\tau_{D} \leq t\right) \geq \frac{1}{1+\left(\frac{a^{2} \pi^{2} \gamma}{2}\right)^{\frac{1}{4}}} \exp \left(-\sqrt{2} a \gamma^{\frac{1}{2}}\right) \\
& \text { if } \sup \{|x-z|: z \in \partial D\} \leq a
\end{aligned}
$$

The lower bound in (1.2) follows from (3.1) and (3.6).

Part ii. By assumption, we can represent $\mu$ in the form $\mu=\mu_{\mathrm{reg}} d x+\mu_{\mathrm{cs}}$, where $\mu_{\text {reg }} \in C_{b}^{2}(D) \cap C^{1}(\bar{D})$ is a sub-probability density on $D$ which coincides with the density $\mu$ in the statement of the theorem in a neighborhood of $\partial D$, and where $\mu_{\mathrm{cs}}$ is a compactly supported sub-probability measure on $D$. By Proposition 1 , we have

$$
\begin{aligned}
\lambda_{0}(\gamma, \mu)= & \gamma \mathcal{E}_{\mu} \exp \left(\left(\lambda_{0}(\gamma, \mu)-\gamma\right) \tau_{D}\right) \\
= & \gamma \int_{D} \mathcal{E}_{x} \exp \left(\left(\lambda_{0}(\gamma, \mu)-\gamma\right) \tau_{D}\right) \mu_{\mathrm{reg}}(x) d x \\
& \quad+\gamma \int_{D} \mathcal{E}_{x} \exp \left(\left(\lambda_{0}(\gamma, \mu)-\gamma\right) \tau_{D}\right) d \mu_{\mathrm{cs}}(x) .
\end{aligned}
$$

The proof of part (iv) showed that

$$
\lim _{\gamma \rightarrow \infty} \gamma \int_{D} \mathcal{E}_{x} \exp \left(\left(\lambda_{0}(\gamma, \mu)-\gamma\right) \tau_{D}\right) d \mu_{\mathrm{cs}}(x)=0 .
$$

Using the fact that close to the boundary $\mu_{\text {reg }}$ coincides with the density $\mu$ in the statement of the theorem along with the assumption that $\mu$ vanishes on the 
boundary, we obtain from (2.5), (2.4) and integration by parts that

$$
\begin{aligned}
& \gamma \int_{D} \mathcal{E}_{x} \exp \left(\left(\lambda_{0}(\gamma, \mu)-\gamma\right) \tau_{D}\right) \mu_{\mathrm{reg}}(x) d x=\gamma \int_{D} u_{\lambda_{0}(\gamma, \mu)-\gamma} \mu_{\mathrm{reg}} d x \\
& =\frac{\gamma}{2\left(\gamma-\lambda_{0}(\gamma, \mu)\right)} \int_{D} \mu_{\mathrm{reg}} \Delta u_{\lambda_{0}(\gamma, \mu)-\gamma} d x \\
& =\frac{\gamma}{2\left(\gamma-\lambda_{0}(\gamma, \mu)\right)} \int_{\partial D} \nabla \mu_{\mathrm{reg}} \cdot n d \sigma+\frac{\gamma}{2\left(\gamma-\lambda_{0}(\gamma, \mu)\right)} \int_{D} u_{\lambda_{0}(\gamma, \mu)-\gamma} \Delta \mu_{\mathrm{reg}} d x
\end{aligned}
$$

where $n$ denotes the inward unit normal on $\partial D$. By assumption, $\Delta \mu_{\text {reg }}$ is bounded in $D$. By Lemma11 and (2.5) it follows that $u_{\lambda_{0}(\gamma, \mu)-\gamma}(x)=\mathcal{E}_{x} \exp \left(\left(\lambda_{0}(\gamma, \mu)-\gamma\right) \tau_{D}\right) \leq$ $\mathcal{E}_{x} \exp \left(-\frac{1}{2} \gamma \tau_{D}\right)$, for sufficiently large $\gamma$. Thus, the bounded convergence theorem and Lemma 1 give

$$
\lim _{\gamma \rightarrow \infty} \frac{\gamma}{2\left(\gamma-\lambda_{0}(\gamma, \mu)\right)} \int_{D} u_{\lambda_{0}(\gamma, \mu)-\gamma} \Delta \mu_{\text {reg }} d x=0 .
$$

From (3.7)-(3.10), Lemma 1 and the fact that $\mu_{\text {reg }}=\mu$ in a neighborhood of $\partial D$, one concludes that

$$
\lim _{\gamma \rightarrow \infty} \lambda_{0}(\gamma, \mu)=\frac{1}{2} \int_{\partial D} \nabla \mu \cdot n d \sigma .
$$

Part iii. From (3.7)-(3.9) and the assumption that $\mu$ and $\nabla \mu$ vanish on $\partial D$, we obtain

$$
\lambda_{0}(\gamma, \mu)=\frac{\gamma}{2\left(\gamma-\lambda_{0}(\gamma, \mu)\right)} \int_{D} u_{\lambda_{0}(\gamma, \mu)-\gamma} \Delta \mu_{\mathrm{reg}} d x
$$

By assumption, $\Delta \mu_{\text {reg }}$ is continuous on $\bar{D}$ and coincides with $\Delta \mu$ in a neighborhood of $\partial D$. Thus, similar to (3.19) in the proof of part (i) below, we have

$$
\lim _{\gamma \rightarrow \infty} \gamma^{\frac{1}{2}} \int_{D} u_{\lambda_{0}(\gamma, \mu)-\gamma} \Delta \mu_{\mathrm{reg}} d x=\frac{1}{\sqrt{2}} \int_{\partial D} \Delta \mu d \sigma
$$

From (3.11), (3.12) and Lemma 1, we conclude that

$$
\lim _{\gamma \rightarrow \infty} \gamma^{\frac{1}{2}} \lambda_{0}(\gamma, \mu)=\frac{1}{2 \sqrt{2}} \int_{\partial D} \Delta \mu d \sigma .
$$

Part i. As the proofs of the other parts have shown, we may ignore any compactly supported part of $\mu$. Thus, by assumption, we may assume that $\mu$ possesses a continuous density, denoted by $\mu$. From (2.6) we have

$$
\lambda_{0}(\gamma, \mu)=\gamma \int_{D} u_{\lambda_{0}(\gamma, \mu)-\gamma} \mu d x
$$

Let $\hat{\mu}$ be the harmonic function in $D$ which coincides with $\mu$ on $\partial D$. Using (2.4), integrating by parts and noting that

$$
\int_{\partial D} u_{\lambda_{0}(\gamma, \mu)-\gamma} \nabla \hat{\mu} \cdot N d \sigma=\int_{\partial D} \nabla \hat{\mu} \cdot N d \sigma=\int_{D} \Delta \hat{u} d x=0,
$$


where $N$ denotes the outward unit normal on $\partial D$, we have

$$
\begin{aligned}
& \int_{D} u_{\lambda_{0}(\gamma, \mu)-\gamma} \hat{\mu} d x=\frac{1}{2\left(\gamma-\lambda_{0}(\gamma, \mu)\right)} \int_{D} \hat{\mu} \Delta u_{\lambda_{0}(\gamma, \mu)-\gamma} d x \\
& =\frac{1}{2\left(\gamma-\lambda_{0}(\gamma, \mu)\right)} \int_{\partial D} \hat{\mu} \nabla u_{\lambda_{0}(\gamma, \mu)-\gamma} \cdot N d \sigma \\
& =\frac{1}{2\left(\gamma-\lambda_{0}(\gamma, \mu)\right)} \int_{\partial D} \mu \nabla u_{\lambda_{0}(\gamma, \mu)-\gamma} \cdot N d \sigma .
\end{aligned}
$$

We will show below that

$$
\lim _{\gamma \rightarrow \infty} \gamma^{-\frac{1}{2}}\left(\nabla u_{-\gamma} \cdot N\right)(x)=\sqrt{2} \text {, uniformly over } x \in \partial D .
$$

From (3.14), (3.15) and Lemma 1 we have

$$
\lim _{\gamma \rightarrow \infty} \gamma^{\frac{1}{2}} \int_{D} u_{\lambda_{0}(\gamma, \mu)-\gamma} \hat{\mu} d x=\frac{1}{\sqrt{2}} \int_{\partial D} \mu d \sigma
$$

Now consider $\gamma^{\frac{1}{2}} \int_{D} u_{\lambda_{0}(\gamma, \mu)-\gamma}(\mu-\hat{\mu}) d x$. The proof of part (iv) showed that $\lim _{\gamma \rightarrow \infty} \gamma^{\frac{1}{2}} \int_{U} u_{\lambda_{0}(\gamma, \mu)-\gamma} d x=0$, for any set $U$ satisfying $\bar{U} \subset D$. Thus, for any $\epsilon>0$,

$$
\begin{aligned}
& \limsup _{\gamma \rightarrow \infty}\left|\gamma^{\frac{1}{2}} \int_{D} u_{\lambda_{0}(\gamma, \mu)-\gamma}(\mu-\hat{\mu}) d x\right| \\
& \leq \sup _{x \in D_{\epsilon}}|\mu(x)-\hat{\mu}(x)| \limsup _{\gamma \rightarrow \infty} \gamma^{\frac{1}{2}} \int_{D} u_{\lambda_{0}(\gamma, \mu)-\gamma} d x,
\end{aligned}
$$

where $D^{\epsilon}$ is as in the statement of the theorem. In the case that $\mu=1$ on $\partial D$, one has $\hat{\mu} \equiv 1$ and (3.16) gives $\lim _{\gamma \rightarrow \infty} \gamma^{\frac{1}{2}} \int_{D} u_{\lambda_{0}(\gamma, \mu)-\gamma} d x=\frac{1}{\sqrt{2}}|\partial D|$. Substituting this in (3.17), using the fact that $\epsilon>0$ is arbitrary and that $\mu$ and $\hat{\mu}$ are continuous and coincide on $\partial D$, we obtain

$$
\lim _{\gamma \rightarrow \infty} \gamma^{\frac{1}{2}} \int_{D} u_{\lambda_{0}(\gamma, \mu)-\gamma}(\mu-\hat{\mu}) d x=0
$$

Now (3.16) and (3.18) give

$$
\lim _{\gamma \rightarrow \infty} \gamma^{\frac{1}{2}} \int_{D} u_{\lambda_{0}(\gamma, \mu)-\gamma} \mu d x=\frac{1}{\sqrt{2}} \int_{\partial D} \mu d \sigma
$$

and thus, from (3.13), we conclude that

$$
\lim _{\gamma \rightarrow \infty} \gamma^{-\frac{1}{2}} \lambda_{0}(\gamma, \mu)=\frac{1}{\sqrt{2}} \int_{\partial D} \mu d \sigma .
$$

We now return to prove (3.15). Fix a point $x_{0} \in \partial D$. We begin with a localization result. For small $\delta>0$, let $(\partial D)^{\delta}=\left\{x \in \partial D: \operatorname{dist}\left(x, x_{0}\right)<\delta\right\}$. Let $U \subset D$ be a domain with $(\partial D)^{\delta} \subset \partial U$. For $\gamma>0$, let $f$ be a continuous function on $\partial U$ satisfying $f(x)=1$, for $x \in(\partial D)^{\delta}$, and $\sup _{x \in \partial U}|f(x)|<\infty$. Let $v_{-\gamma}$ solve the equation

$$
\begin{aligned}
& \frac{1}{2} \Delta v-\gamma v=0 \text { in } U ; \\
& v=f \text { on } \partial U .
\end{aligned}
$$


Let $g_{\gamma}$ denote the restriction of $u_{-\gamma}$ to $\partial U$. Let $W_{\gamma}=u_{-\gamma}-v_{-\gamma}$. Then $W_{\gamma}$ satisfies the equation

$$
\begin{aligned}
& \frac{1}{2} \Delta W-\gamma W=0 \text { in } U \\
& W=g_{\gamma}-f \text { on } \partial U .
\end{aligned}
$$

Let $h(x)$ be a continuous function on $\partial U$ satisfying $h(x) \geq \sup _{\gamma \geq 0}\left|g_{\gamma}(x)-f(x)\right|$, for $x \in \partial U$, and $h(x)=0$, for $x \in(\partial D)^{\delta}$. By the maximum principle,

$$
\left|W_{\gamma}\right| \leq \hat{W}
$$

where $\hat{W}$ satisfies

$$
\begin{aligned}
& \frac{1}{2} \Delta \hat{W}-\gamma \hat{W}=0 \text { in } U ; \\
& \hat{W}=h \text { on } \partial U .
\end{aligned}
$$

Since $W_{\gamma}\left(x_{0}\right)=\hat{W}\left(x_{0}\right)=0$, we have

$$
\left|\left(\nabla W_{\gamma} \cdot N\right)\left(x_{0}\right)\right|=\left|\lim _{n \rightarrow \infty} \frac{W_{\gamma}\left(x_{n}\right)}{\operatorname{dist}\left(x_{n}, x_{0}\right)}\right| \leq \lim _{n \rightarrow \infty} \frac{\hat{W}\left(x_{n}\right)}{\operatorname{dist}\left(x_{n}, x_{0}\right)}=(\nabla \hat{W} \cdot N)\left(x_{0}\right)<\infty,
$$

where $x_{n}$ approaches $x_{0}$ from the normal direction. From (3.22) it follows that

$$
\begin{aligned}
& \lim _{\gamma \rightarrow \infty} \gamma^{-\frac{1}{2}}\left(\nabla u_{-\gamma} \cdot N\right)\left(x_{0}\right)=\sqrt{2} \text { if and only if } \\
& \lim _{\gamma \rightarrow \infty} \gamma^{-\frac{1}{2}}\left(\nabla v_{-\gamma} \cdot N\right)\left(x_{0}\right)=\sqrt{2}, \text { where } v_{-\gamma} \text { solves (3.20). }
\end{aligned}
$$

We now prove a comparison result. Let $D_{i}, i=1,2$, be domains with smooth boundaries satisfying $D_{1} \subset D \subset D_{2}$ and $x_{0} \in \partial D_{i}, i=1,2$. Let $u_{-\gamma}^{(i)}(x)=$ $\mathcal{E}_{x} \exp \left(-\gamma \tau_{D_{i}}\right)$. Either by comparing the stochastic representations or by the maximum principle it follows that $u_{-\gamma}^{(2)}(x) \leq u_{-\gamma}(x) \leq u_{-\gamma}^{(1)}(x)$, for $x \in D^{1}$. Using this along with the fact that $u_{-\gamma}^{(i)}\left(x_{0}\right)=u_{-\gamma}\left(x_{0}\right)=1$, for $i=1,2$, we obtain

$$
\left(\nabla u_{-\gamma}^{(1)} \cdot N\right)\left(x_{0}\right) \leq\left(\nabla u_{-\gamma} \cdot N\right)\left(x_{0}\right) \leq\left(\nabla u_{-\gamma}^{(2)} \cdot N\right)\left(x_{0}\right)
$$

In light of (3.24), to prove (3.15) it is enough to show that if the curvature of $\partial D$ at $x_{0} \in \partial D$ is given by $R \in(-\infty, \infty)$, then

$$
\begin{aligned}
& \lim _{\gamma \rightarrow \infty} \gamma^{-\frac{1}{2}}\left(\nabla u_{-\gamma} \cdot N\right)\left(x_{0}\right)=\sqrt{2}, \text { and the convergence is uniform } \\
& \text { over } R \text { in any bounded set. }
\end{aligned}
$$

In light of this and (3.23), it suffices to consider the following situation: for $R>0$, we consider $\frac{d u_{-\gamma}}{d r}(R)$, where $u_{-\gamma}$ is radially symmetric and satisfies (2.4) and (2.5) with $c=-\gamma$ and $D=A_{\frac{R}{2}, R}(0) \equiv\left\{x \in R^{d}: \frac{R}{2}<|x|<R\right\}$; for $R<0$, we consider $-\frac{d u_{-\gamma}}{d r}(R)$, where $u_{-\gamma}$ is radially symmetric and satisfies (2.4) and (2.5) with $c=-\gamma$, and $D=A_{R, 2 R}(0) \equiv\left\{x \in R^{d}: R<|x|<2 R\right\}$; for $R=0$, the boundary is flat, and without loss of generality we consider $-\frac{d u_{-\gamma}(0)}{d x}$, where $u_{-\gamma}$ satisfies (2.4) and (2.5) with $c=-\gamma$, and $D=(0,1)$.

The flat case has been reduced above to a constant coefficient second-order ODE; one solves explicitly and finds that $\lim _{\gamma \rightarrow \infty} \gamma^{-\frac{1}{2}}\left(-\frac{d u_{-\gamma}(0)}{d x}\right)=\sqrt{2}$. We now turn to 
the case $R>0$. Let

$$
v_{-\gamma}(r)=\phi_{\gamma}(r) \exp (-\sqrt{2 \gamma}(R-r)), \frac{R}{2} \leq r \leq R,
$$

for some function $\phi_{\gamma}$. For a radial function $v(r)$, one has $\Delta v=v^{\prime \prime}+\frac{d-1}{r} v^{\prime}$. Using this with (3.26) one finds that $v_{-\gamma}$ will solve the equation

$$
\begin{aligned}
& \frac{1}{2} \Delta v_{-\gamma}-\gamma v_{-\gamma}=0, \frac{R}{2}<r<R, \\
& v_{-\gamma}(R)=1, v_{-\gamma}^{\prime}(R)=\sqrt{2 \gamma},
\end{aligned}
$$

if $\phi_{\gamma}$ solves the equation

$$
\begin{aligned}
& \frac{1}{2} \phi^{\prime \prime}+\left(\sqrt{2 \gamma}+\frac{d-1}{2 r}\right) \phi^{\prime}+\frac{d-1}{2 r} \sqrt{2 \gamma} \phi=0, \frac{R}{2}<r<R, \\
& \phi(R)=1, \phi^{\prime}(R)=0 .
\end{aligned}
$$

By the standard theory of linear ODE's, (3.28) has a unique solution. Furthermore, one has $\sup _{\gamma>0}\left|\phi_{\gamma}\left(\frac{R}{2}\right)\right|<\infty$. This can be shown by making an appropriate comparison with the solution $\psi_{\gamma}$ of a constant coefficient ODE of the form $\frac{1}{2} \psi^{\prime \prime}+a(\gamma) \psi^{\prime}+b(\gamma) \psi=0$ with $\psi(R)=1$ and $\psi^{\prime}(R)=0$, where $a(\gamma)$ and $b(\gamma)$ are of the order $\gamma^{\frac{1}{2}}$. One can calculate $\psi_{\gamma}$ explicitly and show that $\sup _{\gamma>0}\left|\psi_{\gamma}\left(\frac{R}{2}\right)\right|<\infty$. It follows then that $v_{-\gamma}\left(\frac{R}{2}\right)$ is bounded in $\gamma$. Consequently, the $\left\{v_{-\gamma}\right\}_{\gamma>0}$ solve equations of the form (3.20). (The $f$ in (3.20) will now depend on $\gamma$ but is uniformly bounded in $\gamma$.)

Since $\gamma^{-\frac{1}{2}} \frac{d v_{-\gamma}}{d r}(R)=\sqrt{2}$ and since (3.27) is an equation of the form (3.20), it follows from (3.23) that $\lim _{\gamma \rightarrow \infty} \gamma^{-\frac{1}{2}}\left(\nabla u_{-\gamma} \cdot N\right)(R)=\sqrt{2}$. The difference $\left|\left(\nabla u_{-\gamma} \cdot N\right)(R)-\left(\nabla v_{-\gamma} \cdot N\right)(R)\right|$ is bounded from above by the right hand side of (3.22), where the function $\hat{W}$ solves an equation of the form (3.21) and depends on $R$. By taking the supremum of this quantity over $R$ in a bounded set, one concludes that (3.25) holds. The case $R<0$ is dealt with in an almost identical manner.

\section{Proof of Theorem 2}

We can't use Proposition 11 and (2.6) directly to analyze $\lambda_{0}(\gamma, \mu)$ for small $\gamma$ because $\mathcal{E}_{x} \exp \left(\lambda_{0}^{D} \tau_{D}\right)=\infty$. We make a renormalization. The proof of Proposition 11 showed that $\lambda_{0}(\gamma, \mu)$ solves (2.7) for $\lambda$. Recall the definition of $F_{0}$ in (1.3) and let

$$
V_{\gamma}=w_{\lambda_{0}(\gamma, \mu)-\gamma}-\frac{F_{0} \phi_{0}}{\lambda_{0}^{D}+\gamma-\lambda_{0}(\gamma, \mu)} .
$$

Using (2.1) and the fact that $\phi_{0}$ is the principal eigenfunction corresponding to the principal eigenvalue $\lambda_{0}^{D}$ for the Dirichlet Laplacian, one calculates that

$$
\begin{aligned}
& \frac{1}{2} \Delta V_{\gamma}+\left(\lambda_{0}(\gamma, \mu)-\gamma\right) V_{\gamma}=-1+F_{0} \phi_{0} \text { in } D ; \\
& V_{\gamma}=0 \text { on } \partial D ; \\
& \int_{D} V_{\gamma} \phi_{0} d x=0 .
\end{aligned}
$$

Using (4.1) and recalling the definition of $G_{0}(\mu)$ in (1.3), one can write (2.7) with $\lambda=\lambda_{0}(\gamma, \mu)$ in the form

$$
\gamma \int_{D} V_{\gamma} d \mu+\frac{\gamma F_{0} G_{0}(\mu)}{\lambda_{0}^{D}+\gamma-\lambda_{0}(\gamma, \mu)}=1
$$


or equivalently,

$$
\gamma \int_{D} V_{\gamma} d \mu+\frac{F_{0} G_{0}(\mu)}{1-\frac{\lambda_{0}(\gamma, \mu)-\lambda_{0}^{D}}{\gamma}}=1
$$

From (4.2) we have $\lim _{\gamma \rightarrow 0} V_{\gamma}=V_{0}$, where $V_{0}$ is the solution to (1.4). Thus, it follows from (4.3) that $\lambda_{0}(\gamma, \mu)$ is differentiable from the right at $\gamma=0$ and that $\frac{d^{+} \lambda_{0}}{d \gamma^{+}}(0, \mu)=1-F_{0} G_{0}(\mu)$. In the case that $F_{0} G_{0}(\mu)=1$, it follows from (4.3) that if $\int_{D} V_{0} d \mu<0\left(\int_{D} V_{0} d \mu>0\right)$, then for $\gamma \ll 1$ one has $\lambda_{0}(\gamma, \mu)-\lambda_{0}^{D}>0$ $\left(\lambda_{0}(\gamma, \mu)-\lambda_{0}^{D}<0\right)$.

\section{Proof of Theorem 4}

A number $\lambda \in C$ will constitute an eigenvalue for $L_{\gamma, \mu}$ if and only if there exists a function $u$ vanishing on $\partial D$ satisfying

$$
-\frac{1}{2} \Delta u+\gamma u-\gamma c=\lambda u,
$$

where $c \equiv \int_{D} u d \mu$. (Note that since $L_{\gamma, \mu} u=\lambda u$ and $u \in C_{0}(\bar{D})$, the condition $L_{\gamma, \mu} u \in C_{0}(\bar{D})$ is fulfilled automatically.) Recalling (1.5), the function 1 is represented in $L^{2}(D)$ by $1=\sum_{n=0}^{\infty} F_{n} \phi_{n}$. We represent a proposed eigenfunction $u \in L^{2}(D)$ by $u=\sum_{n=0}^{\infty} c_{n} \phi_{n}$. In order for $u$ to be an eigenfunction, it must lie in the domain of the Dirichlet Laplacian; thus $-\frac{1}{2} \Delta u=\sum_{n=0}^{\infty} \lambda_{n}^{D} c_{n} \phi_{n}$. Substituting in (5.1) and equating coefficients, we find that

$$
c_{n}\left(\gamma+\lambda_{n}^{D}\right)-c \gamma F_{n}=\lambda c_{n}, n=0,1, \ldots
$$

We first show that the condition $E_{\gamma, \mu}(\lambda)=1$ is necessary and sufficient for $\lambda \notin\left\{\gamma+\Lambda_{n}^{D}\right\}_{n=0}^{\infty}$ to be an eigenvalue. Note that if $u$ is an eigenfunction for $L_{\gamma, \mu}$ and $\int_{D} u d \mu=0$, then $u$ is an eigenfunction for $-\frac{1}{2} \Delta$ and thus, for some $n$ and $m$, $u=\phi_{m}, \lambda_{m}^{D}=\Lambda_{n}^{D}$ and $u$ corresponds to the eigenvalue $\gamma+\Lambda_{n}^{D}$. Consequently we may assume that $c \neq 0$. From (5.2), one has

$$
c_{n}=\frac{c \gamma F_{n}}{\lambda_{n}^{D}+\gamma-\lambda}, n=0,1, \ldots
$$

Thus,

$$
u=\sum_{n=0}^{\infty} \frac{c \gamma F_{n}}{\gamma+\lambda_{n}^{D}-\lambda} \phi_{n}
$$

Using the inner product if $\mu$ possesses an $L^{2}$-density, and using Assumption 1 and the bounded convergence theorem otherwise (recall from the remark after Assumption 1 that it always holds if $d=1$ or 2), we have from (5.3) that

$$
\int_{D} u d \mu=\sum_{n=0}^{\infty} \frac{c \gamma F_{n} G_{n}(\mu)}{\gamma+\lambda_{n}^{D}-\lambda}
$$

On the other hand, $0 \neq c=\int_{D} u d \mu$. Thus we conclude from (5.4) that $\lambda \notin$ $\left\{\gamma+\lambda_{n}^{D}\right\}_{n=0}^{\infty}$ is an eigenvalue if and only if

$$
\sum_{n=0}^{\infty} \frac{\gamma F_{n} G_{n}(\mu)}{\gamma+\lambda_{n}^{D}-\lambda}=1 .
$$


Furthermore, it follows that such an eigenvalue is simple, since the corresponding eigenfunction has been uniquely specified (up to a multiplicative constant).

We now consider the possibility that $\lambda=\gamma+\Lambda_{n_{0}}^{D}$ is an eigenvalue, where $n_{0}$ is a nonnegative integer. Let $S_{n_{0}}$ denote the $d_{n_{0}}$-dimensional eigenspace corresponding to the eigenvalue $\Lambda_{n_{0}}$ of the Dirichlet Laplacian. Let $S_{n_{0}}^{G}(\mu)=\left\{v \in S_{n_{0}}: \int_{D} v d \mu=\right.$ $0\}$ and let $S_{n_{0}}^{F}=\left\{v \in S_{n_{0}}: \int_{D} v d x=0\right\}$. Clearly, each of these latter two spaces is either $\left(d_{n_{0}}-1\right)$-dimensional or $d_{n_{0}}$-dimensional.

Consider first the case that $S_{n_{0}}^{F}$ is $\left(d_{n_{0}}-1\right)$-dimensional. There exists an $m_{0}$ such that $\lambda_{m_{0}}^{D}=\Lambda_{n_{0}}^{D}$ and $F_{m_{0}}=\int_{D} \phi_{m_{0}} d x \neq 0$. But then (5.2) will hold with $n=m_{0}$ and $\lambda=\gamma+\Lambda_{n_{0}}^{D}$ if and only if $c=0$. But if $c=0$ and $\lambda=\gamma+\Lambda_{n_{0}}^{D}$, then the argument at the beginning of the second paragraph of the proof forces one to conclude that the eigenfunction $u$ belongs to $S_{n_{0}}^{G}(\mu)$. Consequently, the multiplicity of $\Lambda_{n_{0}}^{D}$ will be either $d_{n_{0}}-1$ or $d_{n_{0}}$, depending on which of these numbers is the dimension of $S_{n_{0}}^{G}(\mu)$. In particular, if $n_{0}=0$, then $d_{n_{0}}=1$ and $S_{n_{0}}^{F}=S_{n_{0}}^{G}(\mu)=\{0\}$ since $\phi_{0}>0$. Thus, $\gamma+\lambda_{0}^{D}=\gamma+\Lambda_{0}^{D}$ can never be an eigenvalue.

Now consider the case that $S_{n_{0}}^{F}$ is $d_{n_{0}}$-dimensional. In this case, $F_{m}=0$, for all $m$ such that $\lambda_{m}^{D}=\Lambda_{n_{0}}^{D}$. We first look for eigenfunctions for which $c \neq 0$. Solving (5.2) gives

$$
\left\{\begin{array}{l}
c_{n}=\frac{c \gamma F_{n}}{\lambda_{n}^{D}+\gamma-\lambda}, \text { for all } n \text { such that } \lambda_{n}^{D} \neq \Lambda_{n_{0}}^{D} \\
c_{n} \text { is arbitrary, for all } n \text { such that } \lambda_{n}^{D}=\Lambda_{n_{0}}^{D}
\end{array} .\right.
$$

Writing $c_{n}=c k_{n}$, for $n$ such that $\lambda_{n}^{D}=\Lambda_{n_{0}}^{D}$, and employing the same reasoning as in (5.3) - (5.5) yields

$$
\sum_{n: \lambda_{n}^{D} \neq \Lambda_{n_{0}}^{D}} \frac{\gamma F_{n} G_{n}(\mu)}{\lambda_{n}^{D}-\Lambda_{n_{0}}^{D}}+\sum_{n: \lambda_{n}^{D}=\Lambda_{n_{0}}^{D}} k_{n} G_{n}(\mu)=1 .
$$

There are two cases to consider: when $S_{n_{0}}^{G}(\mu)$ is $\left(d_{n_{0}}-1\right)$-dimensional and when it is $d_{n_{0}}$-dimensional. In the latter case, $G_{n}(\mu)=0$, for all $n$ satisfying $\lambda_{n}^{D}=\Lambda_{n_{0}}^{D}$. Thus, (5.6) reduces to $E_{\gamma, \mu}\left(\gamma+\Lambda_{n_{0}}^{D}\right)=1$. If this equation is satisfied, we obtain one eigenfunction with $c \neq 0$, and if it is not satisfied, we obtain no such eigenfunctions. Since $S_{n_{0}}^{G}(\mu)$ is $d_{n_{0}}$-dimensional, there are also $d_{n_{0}}$ additional linearly independent eigenfunctions with $c=0$. Thus, the multiplicity is either $d_{n_{0}}+1$ or $d_{n_{0}}$, depending on whether or not $E_{\gamma, \mu}\left(\gamma+\Lambda_{n_{0}}^{D}\right)=1$.

Now consider the case that $S_{n_{0}}^{G}(\mu)$ is $\left(d_{n_{0}}-1\right)$-dimensional. Since we may choose the orthonormal basis $\left\{\phi_{m}\right\}_{\left\{m: \lambda_{m}^{D}=\Lambda_{n_{0}}^{D}\right\}}$ corresponding to the eigenspace $S_{n_{0}}$ however we like, we may assume without loss of generality, that $G_{m}(\mu)=\int_{D} \phi_{m} d \mu=0$, for all but one of the $m$ for which $\lambda_{m}^{D}=\Lambda_{n_{0}}^{D}$. Denote the single $m$ for which this is not true by $m_{0}$. Then (5.6) reduces to

$$
\sum_{n: \lambda_{n}^{D} \neq \Lambda_{n_{0}}^{D}} \frac{\gamma F_{n} G_{n}(\mu)}{\lambda_{n}^{D}-\Lambda_{n_{0}}^{D}}+k_{m_{0}} G_{m_{0}}(\mu)=1 .
$$

The above equation is uniquely solvable for $k_{m_{0}}$ and thus yields one eigenfunction with $c \neq 0$. Since $S_{n_{0}}^{G}(\mu)$ is $\left(d_{n_{0}}-1\right)$-dimensional, there are also $d_{n_{0}}-1$ additional linearly independent eigenfunctions with $c=0$; thus the multiplicity is $d_{n_{0}}$. 


\section{Proof of Theorem 3}

Part i. By Proposition 1 and Theorem 4 it follows that $\lambda_{0}(\gamma, \mu)$ is equal to the smallest root of the equation $E_{\gamma, \mu}(\lambda)=1$, where $E_{\gamma, \mu}$ is as in (1.6). The function 1 has the $L^{2}$-representation $1=\sum_{n=0}^{\infty} F_{n} \phi_{n}$. Since we are assuming that $\mu$ has an $L^{2}$-density, it follows that $\int_{D} 1 d \mu=\sum_{n=0}^{\infty} F_{n} G_{n}(\mu)$; thus,

$$
\sum_{n=0}^{\infty} F_{n} G_{n}(\mu)=\sum_{n=0}^{\infty} \int_{D} P_{\Lambda_{n}^{D}} 1 d \mu=1
$$

First consider the case that $\int_{D} P_{\Lambda_{n}^{D}} 1 d \mu=0$, for all $n \geq 1$. Then it follows from (6.1) that $\int_{D} P_{\Lambda_{0}^{D}} 1 d \mu=1$, and we have $E_{\gamma, \mu}(\lambda)=\frac{\gamma}{\gamma+\lambda_{0}^{D}-\lambda}$. Thus, $E_{\gamma, \mu}\left(\lambda_{0}^{D}\right)=1$, for all $\gamma>0$, and we conclude that $\lambda_{0}(\gamma, \mu)=\lambda_{0}^{D}$, for all $\gamma>0$.

Now consider the case that $\int_{D} P_{\Lambda_{n}^{D}} 1 d \mu \geq 0$, for all $n \geq 1$, and $\int_{D} P_{\Lambda_{n}^{D}} 1 d \mu>0$, for some $n \geq 1$. Recall that $\int_{D} P_{\Lambda_{0}^{D}} 1 d \mu>0$. By Proposition 1 one has $\lambda_{0}(\gamma, \mu)<$ $\gamma+\lambda_{0}^{D}$. To prove that $\lambda_{0}(\gamma, \mu)$ is strictly monotone increasing in $\gamma$, it suffices to show that $E_{\gamma, \mu}(\lambda)$ is increasing as a function of $\lambda \in\left(0, \gamma+\lambda_{0}^{D}\right)$ and that

$$
\left.\frac{d\left(E_{\gamma, \mu}(\lambda)\right)}{d \gamma}\right|_{\lambda=\lambda_{0}(\gamma, \mu)}<0, \text { for } \gamma>0 \text {. }
$$

Trivially, one has $\frac{d E_{\gamma, \mu}(\lambda)}{d \lambda}>0$, for $\lambda \in\left(0, \gamma+\lambda_{0}^{D}\right)$. It remains to show that (6.2) holds.

Differentiating $E_{\gamma, \mu}(\lambda)$ with respect to $\gamma$ gives

$$
\left.\frac{d\left(E_{\gamma, \mu}(\lambda)\right)}{d \gamma}\right|_{\lambda=\lambda_{0}(\gamma, \mu)}=\sum_{n=0}^{\infty} \frac{\left(\Lambda_{n}^{D}-\lambda_{0}(\gamma, \mu)\right) \int_{D} P_{\Lambda_{n}^{D}} 1 d \mu}{\left(\gamma+\Lambda_{n}^{D}-\lambda_{0}(\gamma, \mu)\right)^{2}} .
$$

Subtracting the equation

$$
1=E_{\gamma, \mu}\left(\lambda_{0}(\gamma, \mu)\right)=\sum_{n=0}^{\infty} \frac{\gamma \int_{D} P_{\Lambda_{n}^{D}} 1 d \mu}{\gamma+\Lambda_{n}^{D}-\lambda_{0}(\gamma, \mu)}
$$

from (6.1) gives

$$
\sum_{n=0}^{\infty} \frac{\left(\Lambda_{n}^{D}-\lambda_{0}(\gamma, \mu)\right) \int_{D} P_{\Lambda_{n}^{D}} 1 d \mu}{\gamma+\Lambda_{n}^{D}-\lambda_{0}(\gamma, \mu)}=0
$$

which can be rewritten as

$$
\gamma \sum_{n=0}^{\infty} \frac{\left(\Lambda_{n}^{D}-\lambda_{0}(\gamma, \mu)\right) \int_{D} P_{\Lambda_{n}^{D}} 1 d \mu}{\left(\gamma+\Lambda_{n}^{D}-\lambda_{0}(\gamma, \mu)\right)^{2}}=-\sum_{n=0}^{\infty} \frac{\left(\Lambda_{n}^{D}-\lambda_{0}(\gamma, \mu)\right)^{2} \int_{D} P_{\Lambda_{n}^{D}} 1 d \mu}{\left(\gamma+\Lambda_{n}^{D}-\lambda_{0}(\gamma, \mu)\right)^{2}}
$$

Now (6.2) follows from (6.3), (6.5) and the assumption on $\left\{\int_{D} P_{\Lambda_{n}^{D}} 1 d \mu\right\}_{n=1}^{\infty}$.

We now turn to the case that $\int_{D} P_{\Lambda_{n}^{D}} 1 d \mu \leq 0$, for all $n \geq 1$, and $\int_{D} P_{\Lambda_{n}^{D}} 1 d \mu<0$, for some $n \geq 1$. To prove that $\lambda_{0}(\gamma, \mu)$ is strictly monotone decreasing in $\gamma$, it suffices to show that $E_{\gamma, \mu}(\lambda)$ is increasing as a function of $\lambda \in\left(0, \gamma+\lambda_{0}^{D}\right)$ and that

$$
\left.\frac{d\left(E_{\gamma, \mu}(\lambda)\right)}{d \gamma}\right|_{\lambda=\lambda_{0}(\gamma, \mu)}>0, \text { for } \gamma>0
$$

We have

$$
\frac{d E_{\gamma, \mu}}{d \lambda}(\lambda)=\sum_{n=0}^{\infty} \int_{D} \frac{\gamma P_{\Lambda_{n}^{D}} 1 d \mu}{\left(\gamma+\Lambda_{n}^{D}-\lambda\right)^{2}} .
$$


By (6.1),

$$
\int_{D} P_{\Lambda_{0}^{D}} 1 d \mu>\sum_{n=1}^{\infty}\left(-\int_{D} P_{\Lambda_{n}^{D}} 1 d \mu\right)
$$

Since $\frac{\gamma}{\left(\gamma+\Lambda_{n}^{D}-\lambda\right)^{2}}$ is decreasing in $n$ for $\lambda \in\left(0, \gamma+\Lambda_{0}^{D}\right)$, and since $-\int_{D} P_{\Lambda_{n}^{D}} 1 d \mu \geq 0$, for $n \geq 1$, it follows from (6.8) that

$$
\frac{\gamma}{\left(\gamma+\Lambda_{0}^{D}-\lambda\right)^{2}} \int_{D} P_{\Lambda_{0}^{D}} 1 d \mu>\sum_{n=1}^{\infty} \frac{\gamma}{\left(\gamma+\Lambda_{n}^{D}-\lambda\right)^{2}}\left(-\int_{D} P_{\Lambda_{n}^{D}} 1 d \mu\right)
$$

thus, the right hand side of (6.7) is positive.

The proof of (6.6) is almost identical to the proof of (6.2).

Part ii. By Theorem 4 we must show that the equation $E_{\gamma, \mu}(\lambda)=1$, where $E_{\gamma, \mu}$ is as in (1.6), has no nonreal root $\lambda$. Writing $\lambda=\alpha+i \beta$ and multiplying each summand in $E_{\gamma, \mu}(\alpha+i \beta)$ by the conjugate of its denominator, we have

$$
E_{\gamma, \mu}(\alpha+i \beta)=\sum_{n=0}^{\infty} \frac{\gamma\left(\int_{D} P_{\Lambda_{n}^{D}} 1 d \mu\right)\left(\gamma+\Lambda_{n}^{D}-\alpha+i \beta\right)}{\left(\gamma+\Lambda_{n}^{D}-\alpha\right)^{2}+\beta^{2}} .
$$

Thus, when $\beta \neq 0$, the equation $E_{\gamma, \mu}(\alpha+i \beta)=1$ is equivalent to the equations

$$
\begin{aligned}
& \sum_{n=0}^{\infty} \frac{\gamma \Lambda_{n}^{D}\left(\int_{D} P_{\Lambda_{n}^{D}} 1 d \mu\right)}{\left(\gamma+\Lambda_{n}^{D}-\alpha\right)^{2}+\beta^{2}}=1 ; \\
& \sum_{n=0}^{\infty} \frac{\int_{D} P_{\Lambda_{n}^{D}} 1 d \mu}{\left(\gamma+\Lambda_{n}^{D}-\alpha\right)^{2}+\beta^{2}}=0 .
\end{aligned}
$$

Consider first the case that $\int_{D} P_{\Lambda_{n}^{D}} 1 d \mu \geq 0$, for all $n \geq 1$. Since it is always true that $\int_{D} P_{\Lambda_{0}^{D}} 1 d \mu>0$, the second equation in (6.9) cannot hold. Now consider the case that $\int_{D} P_{\Lambda_{n}^{D}} 1 d \mu \leq 0$ for all $n \geq 1$. If the second equation in (6.9) holds, then one has

$$
\frac{\int_{D} P_{\Lambda_{0}^{D}} 1 d \mu}{\left(\gamma+\Lambda_{0}^{D}-\alpha\right)^{2}+\beta^{2}}=\sum_{n=1}^{\infty} \frac{\left(-\int_{D} P_{\Lambda_{n}^{D}} 1 d \mu\right)}{\left(\gamma+\Lambda_{n}^{D}-\alpha\right)^{2}+\beta^{2}} .
$$

Using this and the fact that $\Lambda_{n}^{D}$ is increasing in $n$, we obtain

$$
\frac{\gamma \Lambda_{0}^{D} \int_{D} P_{\Lambda_{0}^{D}} 1 d \mu}{\left(\gamma+\Lambda_{0}^{D}-\alpha\right)^{2}+\beta^{2}}<\sum_{n=1}^{\infty} \frac{\gamma \Lambda_{n}^{D}\left(-\int_{D} P_{\Lambda_{n}^{D}} 1 d \mu\right)}{\left(\gamma+\Lambda_{n}^{D}-\alpha\right)^{2}+\beta^{2}}
$$

thus the first equation in (6.9) cannot hold.

Part iii. By assumption, all of the eigenvalues $\left\{\lambda_{n}^{D}\right\}_{n=0}^{\infty}$ are distinct, and $F_{n} G_{n}(\mu)>0$, for all $n$; thus, it follows from Theorem 4 that the set of eigenvalues of $L_{\gamma, \mu}$ coincides with the set of roots $\lambda$ of the equation $E_{\gamma, \mu}(\lambda)=1$. The condition $F_{n} G_{n}(\mu)>0$, for all $n$, guarantees that $E_{\gamma, \mu}(\lambda)$ is increasing for $\lambda \in\left(0, \gamma+\lambda_{0}^{D}\right)$ and satisfies $E_{\gamma, \mu}(0)<1$ and $E_{\gamma, \mu}\left(\left(\gamma+\lambda_{0}^{D}\right)^{-}\right)=\infty$, and that for each $n \geq 0, E_{\gamma, \mu}(\lambda)$ is increasing for $\lambda \in\left(\gamma+\lambda_{n}^{D}, \gamma+\lambda_{n+1}^{D}\right)$ and satisfies $E_{\gamma, \mu}\left(\left(\gamma+\lambda_{n}^{D}\right)^{+}\right)=-\infty$ and $E_{\gamma, \mu}\left(\left(\gamma+\lambda_{n+1}^{D}\right)^{-}\right)=\infty$. Thus, there is exactly one root between 0 and $\gamma+\lambda_{0}^{D}$ and exactly one root between $\gamma+\lambda_{n}^{D}$ and $\gamma+\lambda_{n+1}^{D}$, for $n \geq 0$. Consequently, $\gamma+\lambda_{n-1}^{D}<\lambda_{n}(\gamma, \mu)<\gamma+\lambda_{n}^{D}$, for $n \geq 1$. 


\section{Appendix A. The Semigroup $T_{t}^{\gamma, \mu}$ And its COnnection to $L_{\gamma, \mu}$}

We first show that $-L_{\gamma, \mu}$, defined on $C^{2}(\bar{D}) \cap\left\{u: u, L_{\gamma, \mu} u \in C_{0}(\bar{D})\right\}$, and then extended appropriately, is the infinitesimal generator of $T_{t}^{\gamma, \mu}$. From the definition of the Brownian motion with random jumps we have

$$
\begin{aligned}
& T_{t}^{\gamma, \mu} u(x)=\exp (-\gamma t) \int_{D} p^{D}(t, x, y) u(y) d y \\
& +\int_{0}^{t} \gamma \exp (-\gamma s) \int_{D} p^{D}(t-s, \mu, y) u(y) d y d s,
\end{aligned}
$$

where $p^{D}(t, x, y)$ is the transition sub-probability density for Brownian motion in $D$ starting at $x \in D$ and killed at the boundary. A standard result for the semigroup corresponding to Brownian motion (without jumps) allows us to conclude that for $u \in C^{2}(\bar{D}) \cap C_{0}(\bar{D})$, one has $\lim _{t \rightarrow 0} \frac{\int_{D} p^{D}(t, x, y) u(y) d y-u(x)}{t}=\frac{1}{2}(\Delta u)(x)$, and the convergence is uniform over $x \in \bar{D}$. Using this and (A.1), we obtain $\lim _{t \rightarrow 0} \frac{T_{t}^{\gamma, \mu} u(x)-u(x)}{t}=\frac{1}{2}(\Delta u)(x)-\gamma u(x)+\gamma \int_{D} u d \mu=-\left(L_{\gamma, \mu} u\right)(x)$, and the convergence is uniform over $x \in \bar{D}$. Furthermore, by assumption, $-L_{\gamma, \mu} u \in C_{0}(\bar{D})$. Thus, the infinitesimal generator of $T_{t}^{\gamma, \mu}$ is indeed an extension of $-L_{\gamma, \mu}$ on $C^{2}(\bar{D}) \cap$ $\left\{u: u, L_{\gamma, \mu} u \in C_{0}(\bar{D})\right\}$.

We now turn to compactness.

Proposition 2. The semigroup $T_{t}^{\gamma, \mu}$ is compact.

Proof. Let $\left\{Z_{n}\right\}_{n=1}^{\infty}$ be a sequence of IID random variables distributed according to the exponential distribution with parameter $\gamma$, and let $S_{n}=\sum_{j=1}^{n} Z_{j}$. Denote probabilities for these random variables by $Q$. From the definition of the Brownian motion with jumps, it follows that

$$
\begin{aligned}
& T_{t}^{\gamma, \mu} f(x)=\exp (-\gamma t) \int_{D} p^{D}(t, x, y) f(y) d y \\
& +\sum_{n=1}^{\infty} \int_{0}^{t} \int_{D} p^{D}(t-s, \mu, y) f(y) Q\left(S_{n}=s, S_{n+1}>t\right) d y d s \\
& \equiv \exp (-\gamma t) \int_{D} p^{D}(t, x, y) f(y) d y+T_{t}^{\gamma, \mu ; \infty} f(x) .
\end{aligned}
$$

It is known [6] that for any $\epsilon>0, p^{D}(t, x, y)$ is continuous on $[\epsilon, \infty) \times \bar{D} \times \bar{D}$, and thus uniformly continuous on $[\epsilon, T] \times \bar{D} \times \bar{D}$, for $0<\epsilon<T<\infty$. Thus the transformation $f(x) \rightarrow \exp (-\gamma t) \int_{D} p^{D}(t, x, y) f(y) d y$ maps bounded sets in $C_{0}(\bar{D})$ to equicontinuous and bounded sets in $C_{0}(\bar{D})$; consequently, this transformation is compact. It remains to show that $T_{t}^{\gamma, \mu ; \infty}$ is a compact map.

For $m \geq 1$, define the map $T_{t}^{\gamma, \mu ; m}$ by

$$
T_{t}^{\gamma, \mu ; m} f(x)=\sum_{n=1}^{m} \int_{0}^{t} \int_{D} p^{D}(t-s, \mu, y) f(y) Q\left(S_{n}=s, S_{n+1}>t\right) d y d s .
$$

Then we have $\left\|\mid T_{t}^{\gamma, \mu ; \infty}-T_{t}^{\gamma, \mu ; m}\right\| \leq \sum_{n=m+1}^{\infty} Q\left(S_{n} \leq t, S_{n+1}>t\right)=Q\left(S_{m+1} \leq t\right)$. Thus, $T_{t}^{\gamma, \mu ; m}$ converges in the operator norm to $T_{t}^{\gamma, \mu ; \infty}$. Consequently, we need only show that the map $U_{n}$, defined by

$$
U_{n} f(x)=\int_{0}^{t} \int_{D} p^{D}(t-s, \mu, y) f(y) Q\left(S_{n}=s, S_{n+1}>t\right) d y d s,
$$


is compact. Define the map $U_{n, \epsilon}$ by

$$
U_{n, \epsilon} f(x)=\int_{0}^{t-\epsilon} \int_{D} p^{D}(t-s, \mu, y) f(y) Q\left(S_{n}=s, S_{n+1}>t\right) d y d s
$$

Then $\left\|U_{n}-U_{n, \epsilon}\right\| \leq Q\left(S_{n} \in[t-\epsilon, t]\right)$. Thus, $U_{n, \epsilon}$ converges to $U_{n}$ in the operator norm and consequently it suffices to show that $U_{n . \epsilon}$ is compact. By the above noted uniform continuity of $p^{D}(t, x, y), U_{n, \epsilon}$ maps bounded sets in $C_{0}(\bar{D})$ to equicontinuous and bounded sets in $C_{0}(\bar{D})$; consequently it is compact.

\section{REFERENCES}

[1] Ben-Ari, I. and Pinsky, R. G., Ergodic behavior of diffusions with random jumps from the boundary, Stochastic Processes and their Applications, to appear.

[2] Ben-Ari, I. and Pinsky, R. G., Spectral analysis of a family of second-order elliptic operators with nonlocal boundary condition indexed by a probability measure, J. Funct. Anal. 251 (2007), 122-140. MR2353702

[3] Davidson, F. A. and Dodds, N. Spectral properties of non-local uniformly-elliptic operators, Electron. J. Differential Equations (2006) No. 126, 15 pp. (electronic). MR 2255241 (2007e:35046)

[4] Freitas, P., A nonlocal Sturm-Liouville eigenvalue problem, Proc. Roy. Soc. Edinburgh Sect. A 124 (1994), 169-188. MR1272438 (95b:34036)

[5] Freitas, P. and Vishnevskii, M. P., Stability of stationary solutions of nonlocal reactiondiffusion equations in $m$-dimensional space, Differential Integral Equations 13 (2000), 265288. MR1811959 (2001m:35173)

[6] Friedman, A. Partial Differential Equations of Parabolic Type, Prentice-Hall, Inc., Englewood Cliffs, N.J., (1964). MR0181836 (31:6062)

[7] Grieser, D., Uniform bounds for eigenfunctions of the Laplacian on manifolds with boundary, Comm. Partial Differential Equations 27 (2002), 1283-1299. MR1924468(2003g:58036)

[8] Grigorescu, I. and Kang, M., Brownian motion on the figure eight, J. Theoret. Probab. 15, (2002), 817-844. MR1922448 (2003f:60144)

[9] Grigorescu, I. and Kang, M., Ergodic properties of multidimensional Brownian motion with rebirth, preprint, url: http://www.math.miami.edu/ igrigore/pp/gn.pdf.

[10] Karatzas, I. and Shreve, S., Brownian Motion and Stochastic Calculus, Springer-Verlag, New York, 1988. MR.917065 (89c:60096)

[11] Leung, Y., Li, W. and Rakesh, Spectral analysis of Brownian motion with jump boundary, Proc. Amer. Math. Soc., 136 (2008), no. 12, 4427-4436. MR2431059

[12] Pazy, A., Semigroups of Linear Operators and Applications to Partial Differential Equations, Applied Mathematical Sciences, 44, Springer-Verlag, New York, (1983). MR710486 (85g:47061)

[13] Pinsky, R. G., The dead core for reaction-diffusion equations with convection and its connection with the first exit time of the related Markov diffusion process, Nonlinear Anal. 12, (1988), 451-471. MR940604 (89h:35161)

[14] Pinsky, R. G., Positive Harmonic Functions and Diffusion, Cambridge Studies in Advanced Mathematics 45, Cambridge University Press, (1995). MR.1326606 (96m:60179)

[15] Reed, M. and Simon, B., Methods of Modern Mathematical Physics, I, Functional Analysis, Academic Press, New York, (1972). MR0493419 (58:12429a)

Department of Mathematics, Technion-Israel Institute of Technology, Haifa, 32000, ISRAEL

E-mail address: pinsky@math.technion.ac.il

URL: http://www.math.technion.ac.il/〜pinsky/ 